\title{
Disruption handling in large railway networks
}

\author{
F. Corman ${ }^{1}$, A. D’ Ariano ${ }^{2}$ \& I. A. Hansen ${ }^{1}$ \\ ${ }^{1}$ Department of Transport and Planning, \\ Delft University of Technology, The Netherlands \\ ${ }^{2}$ Dipartimento di Informatica e Automazione, \\ Università degli Studi Roma Tre, Italy
}

\begin{abstract}
Railway traffic is operated according to a detailed off-line plan of operations, specifying for each train its path through the network plus arrival and departure times at its scheduled stops. During daily operations disturbances perturb the plan and dispatchers take actions in order to keep operations feasible and to limit delay propagation. This paper studies how to support dispatchers in the management of infrastructure disruptions in a large network divided into dispatching areas of limited size. We consider seriously disturbed traffic conditions on double track railway lines where some block sections of one track are unavailable for traffic, e.g., due to a temporary track blockage. We start from a given set of disruption resolution scenarios, computed off-line. Disrupted train services are either cancelled, rerouted in the disrupted dispatching area or rerouted in other areas while still with the same origin and destination. Given a disruption resolution scenario, we adopt an advanced decision support system, ROMA (Railway traffic Optimization by Means of Alternative graphs), in order to compute efficient train schedules at the level of signal control, along with detailed performance indicators. Alternative dispatching solutions are computed in a few minutes. The dispatcher has to choose one schedule for implementation, with a trade-off between minimizing delays of all trains running in the network and limiting the cancellation of disrupted train services. A test case is evaluated on a large Dutch railway network with heavy traffic and strong disorder. We compute dispatching solutions for a given set of scenarios and evaluate them in terms of travel times, waiting times and train delays. Keywords: railway traffic management, disruption, microsimulation.
\end{abstract}




\section{Introduction}

Train services are planned in detail, defining several months in advance the train order and timing at crossings, junctions and platform tracks. A robust timetable is able to deal with minor perturbations (i.e., few minutes of delays) occurring in real-time by using smart planning rules and time reserves. However, no reasonable railway plan is robust or reliable enough in the case of large delays or the blocking of some tracks. Despite the great effort spent, technical failures and other disturbances (such as train delays, reduced operating speeds, bad weather and temporary unavailability of some routes) can be considered as disruptions [2], since they may influence seriously the running times, dwelling and departing events of trains. In fact, trains may be required to stop in front of crossings or junctions, causing non-scheduled waiting times and longer running times due to slowing down and subsequent re-acceleration. Due to the interaction between trains, such disturbances can propagate as knock-on delays to other trains in the network. Hence, the railway system is vulnerable to disruptions and railway traffic has to be rescheduled in real-time. The management of a disruption requires the modification of train services, making alterations to the train travel times and routes due to the temporary unavailability of one or more block sections. Multiple timetable modifications are thus required in order to recover the feasibility of disrupted operations.

Operational traffic management is mainly directed towards recovering from disruptive events as quickly as possible. The train operating companies adjust, in a short time, the personnel and rolling stock plan in order to be compliant with the actual traffic situation, avoiding imbalances that may result in the unavailability of train units or crews. The dispatchers then manage the train traffic under shortage of spare capacity and several delayed trains. Experienced dispatchers have developed strategies allowing them simply to foresee possible disruptions well in advance and to take compensatory control actions based on local information. Delays are experienced in a first instance by the passengers and goods being transported. However, dispatchers only reschedule the route setting plan when trains have a considerable delay, and become active only when train traffic is already highly disrupted.

In The Netherlands, so called emergency timetables are used as a response to disrupted operations. The dispatchers apply predetermined if-then measures in order to determine an alternative train path until the next station and keep the (network) traffic control center informed. The intention is to limit modifications of the rolling stock and crew plan as much as possible. However, in case of disruptions some of the original services can be cancelled or some trains can be rerouted. Emergency timetables cover every possible infrastructure malfunction. As the variety of possible disruptions is very large, some of them are grouped together in classes, so that the amount of emergency timetable is limited (now there are around 1200 emergency timetables). Figure 1 shows a practical example of emergency timetable. The disruption under discussion is reported graphically in terms of the infrastructure unavailability, namely a block on the upper track from 


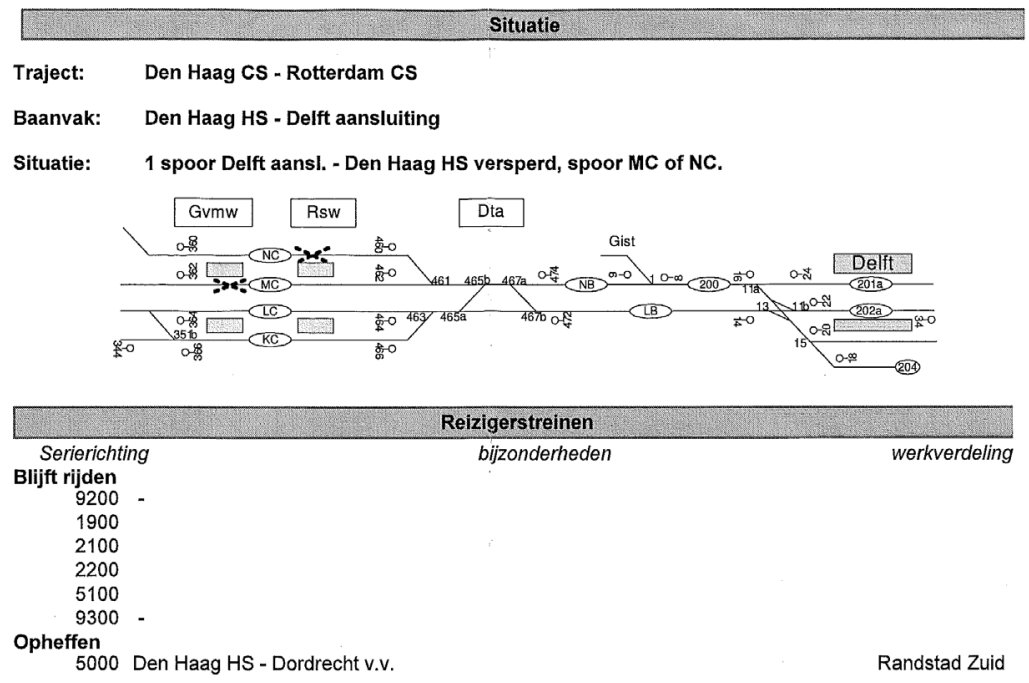

Figure 1: Example of emergency timetable in The Netherlands (source: ProRail).

Delft (right) to The Hague (Gvmw, left). The modifications to the services are reported on the lower part of the figure (here, the Dutch words 'blijft rijden' mean that the service is kept running, while 'opheffen' means that the service 5000 is cancelled between the stations of Den Haag HS and Dordrecht).

Post-eventum analysis is used to analyze the causes of disturbances and to compute pro-actively emergency timetables. In this context, Wiklund [13] proposes a useful tool to analyze disruption handling strategies in Sweden. A framework is given to quantify the effect of disturbances by looking at simple indicators like the amount of traffic mileage that is actually accomplished in the timetable put into operations. Simulation tests analyze different timetables for application during a serious disruption due to adverse weather conditions.

With regards to the recent literature on rolling stock balancing and crew scheduling, Jespersen-Groth et al. [9] present a detailed report on models and procedures used to manage disruptions in Denmark and in The Netherlands. Nielsen et al. [10] also address rescheduling of railway crew plans when these are unfeasible due to disrupted traffic. An online setup solves iteratively a linear programming model for crew assignment in limited time horizon. The procedure is repeated when new data about the expected disruption duration is collected. The main assumption is that a feasible train timetable for the disrupted operations exists when a solution to crew rescheduling is computed.

Concerning train rescheduling during disrupted operations, Hirai et al. [8] propose a train dispatching algorithm with a train rescheduling pattern language processing system. In case of severe traffic disruptions, caused by accidents that may require the suspension of some train line, the algorithm is helpful for the preparation of practical rescheduling plans. Based on actual train schedule data of a 
Japanese railway network, the authors report that the approach works satisfactorily even if only local modifications are applied to the original schedule.

Differently from the previous approach, Takagi et al. [11] perform numerical optimization on a rail junction when the service is disrupted. In the optimization routine, a genetic algorithm is used to improve the order of route setting, i.e., to minimize a weighted sum of times. When a route setting decision has been taken, the signaling system is automatically set. The object-oriented multi-train simulator has been tested on metro lines and suburban lines.

In the current traffic control practice, dispatchers have a limited view on the possible actions to undertake, and the existing dispatching systems are only used to monitor a disrupted situation rather than to control train traffic. A number of decision support tools for optimal dispatching in perturbed traffic conditions have been proposed so far (see the literature reviews in [5, 12]). However, the dispatching practice still requires further development of Decision Support Systems (DSS) that are able to compute dispatching solutions for large networks and serious disruptions at the level of signal control. This is the main reason for the research proposed in this paper. Furthermore, the dispatching process is inherently multi objective, since the rescheduling solutions should match the interest of the different actors involved (dispatchers, train operating companies and passengers). Decisions are therefore to be supported by a clear vision of the future status of the network, so that dispatchers can justify quantitatively their decisions against the conflicting goals of the multiple actors in the railway business. In addition, managing disrupted traffic requires substantial timetable modifications in terms of train orders, times and routes, or even cancellation of some train services.

This paper studies how to support dispatchers in the management of infrastructure disruptions in a large network. Section 2 describes an innovative DSS to handle disrupted traffic in large networks. This system, called ROMA (Railway traffic Optimization by Means of Alternative graphs) [5, 6], is designed to assist dispatchers in their tasks. ROMA is able to estimate and control the future evolution of the railway traffic by considering actual track occupation, signaling and safety constraints, as well as dynamic train characteristics. The feasibility of train movements is checked by estimation of microscopic blocking times (see, e.g., the blocking time theory described in the book of Hansen and Pachl [7]), while the resolution of potential train conflicts and/or deadlocks is based on optimal computation of train routes, orders and target speeds. The objective is to compute a new feasible plan of operations (emergency timetable) for a given set of disruption resolution scenarios, limiting the propagation of train delays. The relative improvement of ROMA compared to practical dispatching rules is reported e.g., in [3-6].

Section 3 presents a practical test case based on a large Dutch railway network. Given disruptions such as unavailability of tracks and limited performance of some train units, we use ROMA to study different alternative disruption resolution scenarios. In Section 4, performance indicators are given for a number of dispatching solutions, one for each scenario, so that the dispatcher can take an informed decision based on a quantitative evaluation of their effects. 


\section{Disruption handling procedure}

We analyze disruptions (such as a train malfunction or an infrastructure failure) on double track railway lines, where some block sections of one track are unavailable for traffic. The available capacity experiences a strong reduction for the long period of time, from a few hours and up to days, that is required to restore the infrastructure availability. The time reserves in the timetable are not sufficient to prevent delay propagation and a new plan of operations is needed to allow feasible train circulation under the very limited capacity.

The disruption handling procedure considers a given set of disruption resolution scenarios as input. A disruption resolution scenario is a serious modification of the original timetable. Disrupted train services are either cancelled (when trains are short-turned in the vicinity of a disruption and directed towards a shunting area), rerouted in the disrupted dispatching area (using a locally available undisrupted track) or rerouted in other areas while still maintaining the same origin and destination (when there is at least a possibility to bypass a disruption via other train routes). For each disruption resolution scenario, we assume that feasible rolling stock and crew management plans are available. In other words, we recover feasibility of operations by a sequential approach with respect to the rolling stock, crew and train services. The first level decision is the selection of a set of disruption resolution scenarios, while the second level decision is the implementation of a chosen scenario. For the latter decision, we compute a new timetable such all trains run safely in the network, minimizing the propagation of delays in operation.

The approach to the whole disruption handling problem is presented schematically in Figure 2. A set of disruption resolution scenarios is supplied as input to the management of a large railway network. We adopt ROMA in order to compute a feasible plan of operations for each scenario, i.e., the train schedule is compliant with the railway operating rules of and with the entrance position of each train.

The railway traffic management problem is solved by considering a network division into local areas of limited size. As shown in Figure 2, each local

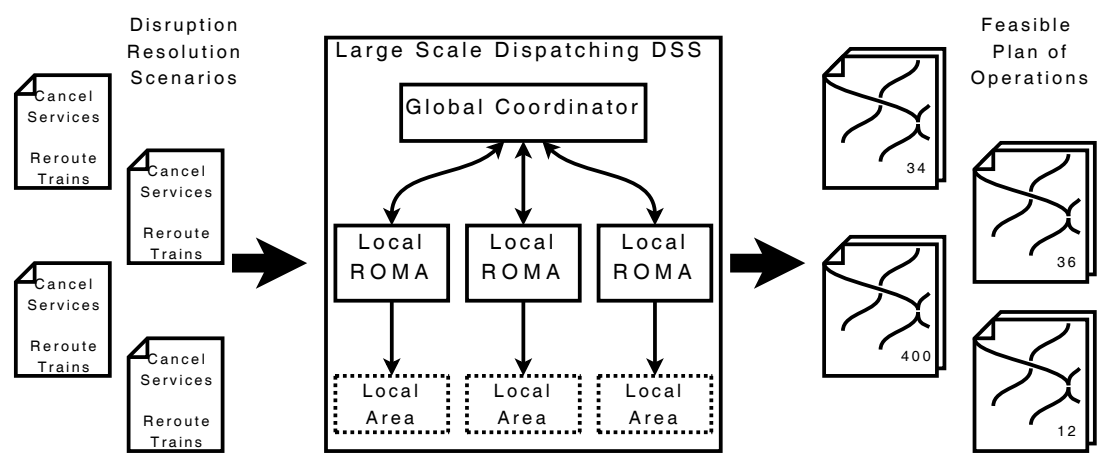

Figure 2: Architecture of the multi-area disruption handling approach. 
area is controlled by a local scheduler (that gives support to the local human dispatcher) while the overall area is supervised by a coordination system at a higher level. The global coordinator is asked to repair the situation when there is an infeasibility involving two or more areas. The feasibility of operations is recovered by imposing additional constraints to the local schedulers and by computing new local solutions. In this paper, we use the coordination procedure of Corman et al. [3] and the local train scheduling procedure of D'Ariano et al. [4]. The output of the DSS consists in a set of feasible plans of operations, one for each disruption resolution scenario, that are represented by time distance graphs and detailed performance indicators. The local dispatcher has thus to choose a single train schedule for implementation, with least train delays and cancelled train services.

\section{Test case description}

This section presents a real-world test case based on a large part of the Dutch railway network, including the main stations of Den Bosch, Nijmegen, Arnhem and Utrecht. The railway network under discussion is mostly double tracked. Figure 3 shows the network division into three local dispatching areas.

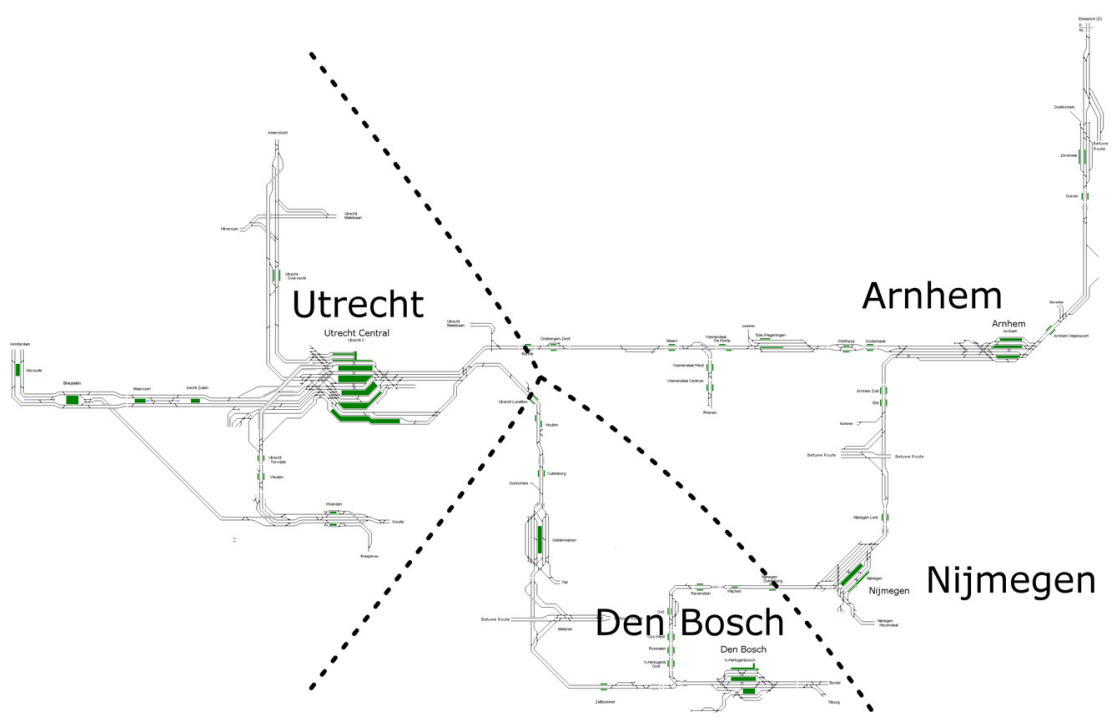

Figure 3: The railway network divided into three local dispatching areas.

The general network layout comprises a diameter of about $100 \mathrm{~km}$. In total, there are more than 1200 block sections and stopping platforms at stations. The two main traffic directions are served by the line between Utrecht and Arnhem (towards Germany) and the line between Utrecht and Den Bosch (from Amsterdam 
towards Eindhoven and the southern part of the country). The reference timetable is periodic with a cycle time of one hour that schedules around 150 trains.

We consider a disruption located on the line Utrecht - Den Bosch (near Zaltbommel) that blocks one track and reduces the maximum train speed allowed on the adjacent track $(60 \mathrm{~km} / \mathrm{h}$ instead of $130 \mathrm{~km} / \mathrm{h})$. Trains of both traffic directions have to run on the single track and under the constraints on the maximum allowed speed for a stretch of around $6 \mathrm{~km}$. In the original timetable, 12 trains per hour (6 per direction) are scheduled on the disrupted line.

In Figure 4, we report the hourly timetable and five alternative disruption resolution scenarios. Every solid line indicates that there are two trains running
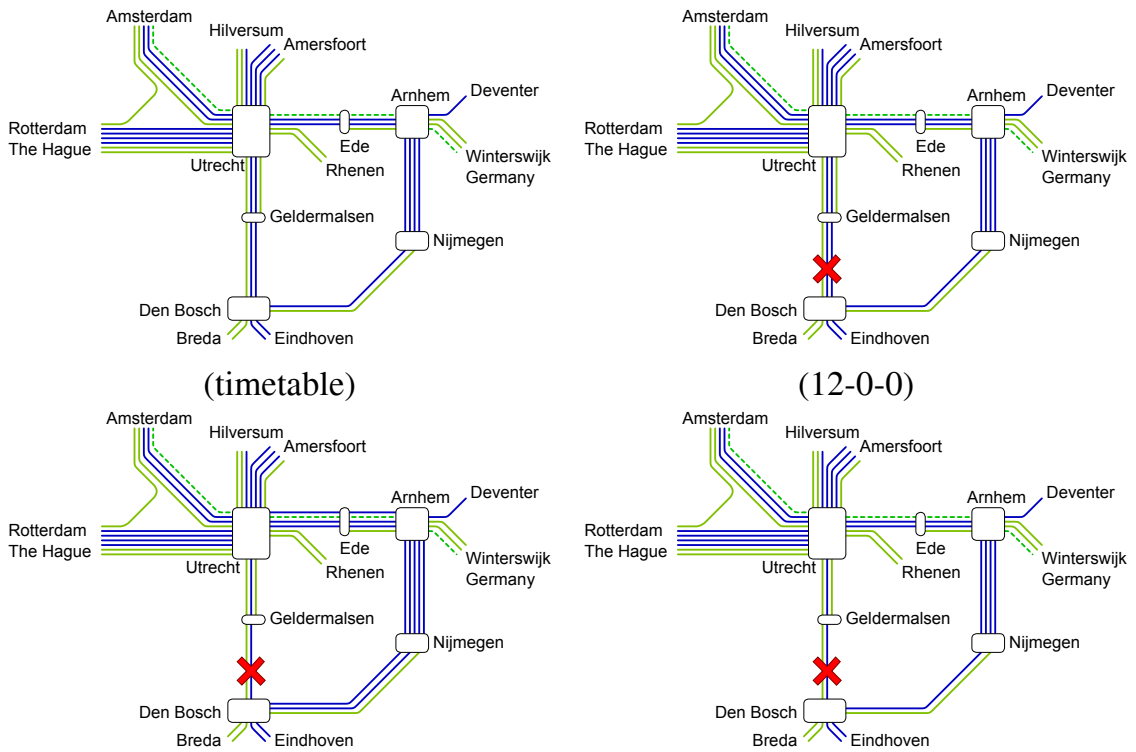

(8-4-0)

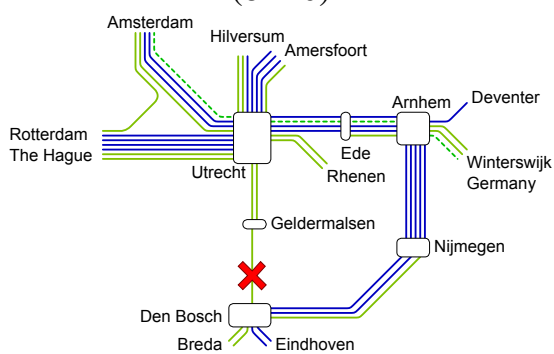

(4-4-4)

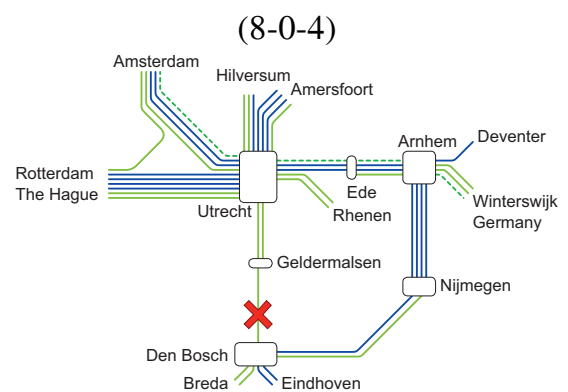

(4-0-8)

Figure 4: Available train services for the timetable and for each scenario (color online only). 
per hour per direction on a specific line. Light green lines are local services and dark blue lines are intercity services. The dotted line represents an international service scheduled once per hour. The track blockage is represented by the cross between the stations of Geldermalsen and Den Bosch. The disruption resolution scenarios are defined by the three field code $A-B-C$, where $A$ is the number of trains locally rerouted to bypass the disruption, $B$ is the number of trains globally rerouted via Arnhem and Nijmegen, and $C$ is the number of train services cancelled.

12-0-0 All the trains are scheduled as in the timetable. The only difference is that the line Utrecht - Den Bosch is served by twelve trains (8 intercity and 4 local trains) per hour running on the same single track.

8-4-0 Four intercity trains ( 2 trains per hour per direction) and four local trains (2 trains per hour per direction) are still scheduled on the line Utrecht Den Bosch. The other four intercity trains ( 2 trains per hour per direction) are globally rerouted, i.e., change their routes but keep the same origin and destination stations. The latter trains are rerouted via the line going to Nijmegen and Arnhem, or vice versa. The running time required for the alternative trip between Utrecht and Den Bosch is around 40 minutes longer than the original trip time, which is 30 minutes long.

8-0-4 Here, four intercity and four local trains are scheduled on the line Utrecht - Den Bosch, while the other four intercity train services are cancelled, resulting in less trains running in the railway network. The corresponding trains are thus held in the major stations of Utrecht and Den Bosch, assuming that there is enough spare capacity at stations yards.

4-4-4 Four local trains are still scheduled on the line Utrecht - Den Bosch, four intercity trains are globally rerouted via the line Nijmegen - Arnhem and four intercity trains are held in the major stations of Utrecht and Den Bosch.

4-0-8 Four local trains are still scheduled on the line Utrecht - Den Bosch while eight intercity trains are held in the major stations of Utrecht and Den Bosch. The local trains thus serve all passengers.

\section{Computational results}

This section presents the results obtained by ROMA within 10 minutes of computation on an Intel Core Duo $1.66 \mathrm{Ghz}$ workstation with 2GB RAM. For each disruption resolution scenario, Figure 5 reports the blocking time diagram in the vicinity of the studied disruption. The diagram on the top left side also shows the original traffic on the same line in absence of disruptions (i.e., the timetable). This figure focuses on the first 45 minutes of traffic prediction (see the y-axis), since these are most interesting to understand the short-term effects of disruption handling. Each blocking time diagram shows the train traffic on the line Den Bosch - Utrecht, that is around 40 kilometers long (see the x-axis). The following stations are considered from left to right: Den Bosch $(\mathrm{Ht})$, Zaltbommel (Zbm), Geldermalsen (Gdm), Culemborg $(\mathrm{Cl})$, Houten ( $\mathrm{Htn})$ and Utrecht Lunetten (Utl). 


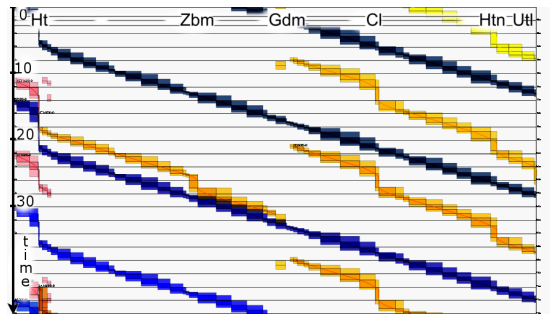

(timetable)

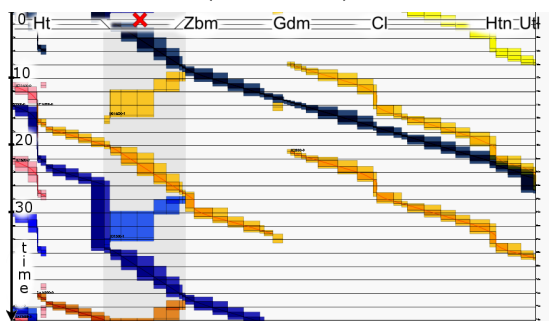

(8-4-0)

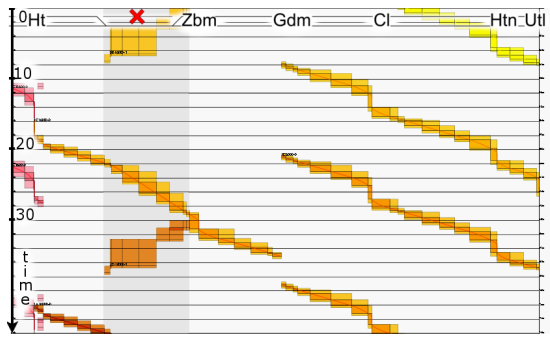

(4-4-4)

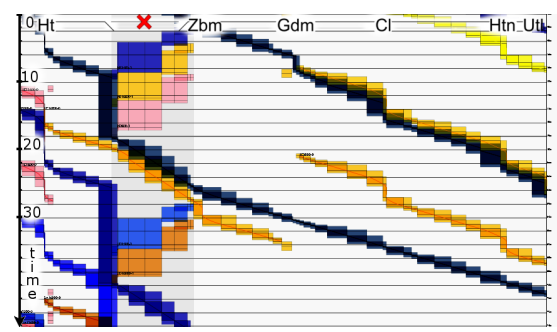

(12-0-0)

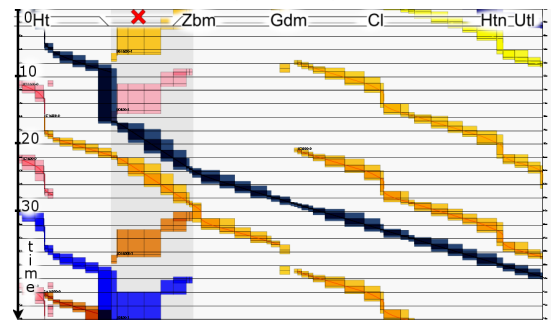

$(8-0-4)$

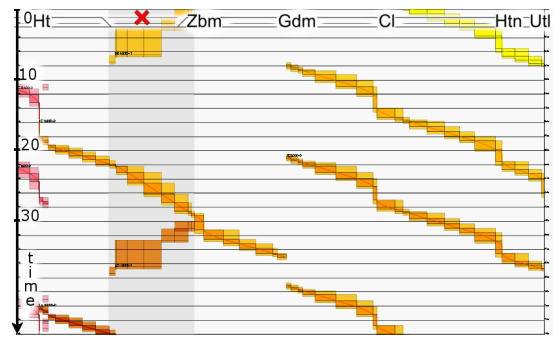

(4-0-8)

Figure 5: Blocking time diagrams between Den Bosch and Utrecht Lunetten.

The blocking time diagrams of Figure 5 can be adopted as support to dispatchers when solving disrupted traffic situations. Comparing the five scenarios, dispatchers can easily detect the delayed trains by observing the long stretched blocking times, which represent trains waiting in front of a red signal in order to enter the single track area. The available spare capacity can be measured as the time lag between blocking time graphs of two consecutive trains on a block section. When there is no spare capacity between the passage of two trains on a block section (i.e., the corresponding time lag is null) the following train may reduce its speed according to the signalling system in use.

We next report the performance indicators that are used in this paper in order to assess the quality of each feasible plan of operations for a given scenario:

- The travel time reports the time required by the passengers on a train to travel on a specific corridor. Given two stations delimiting a corridor, this is the travel time between the first and the last station. 
- The waiting time is another indicator related to passengers' satisfaction. This time is computed as the average time between two consecutive departure times at a given station for trains travelling over a given corridor. For a period of traffic prediction including $n$ trains, the average waiting time is $\sum_{i=1}^{n}\left[t_{A}^{(i+1)}-t_{A}^{i}\right] / n$, where $i$ and $i+1$ are two consecutive trains departing from the station $A$ and running on the same corridor.

- The generalized travel time is a weighted sum of the two latter indicators. Given an origin-destination pair, we define this time as $2 *$ waiting time + travel time. In this case, we assume that passengers penalize more a long waiting time at a station rather than a long travel time, as described in [1].

- The minimum cycle time is the time required to dispatch all the scheduled services. We observe that if this time is longer than one hour, there is not enough capacity in order to include all trains.

- The track occupancy is another measure of the capacity required to dispatch all the scheduled services. This is computed as the ratio between the minimal time required to run all the trains, without including time reserves, and the actual plan of operations.

- The output delay is the train delay at its exit from the network. In our experiments the output delays are originated by the disrupted track between Geldermalsen and Den Bosch, while there are no input delays, i.e., the initial position of all trains corresponds to the one in the timetable.

Table 1 reports the results for the reference case with no disruption (i.e., the timetable that is no more feasible, see the first row of the table) and for the alternative solutions in presence of disruption (i.e., the feasible train schedules computed for each scenario, see the other rows of the table). The indicators described above are reported in the table for each plan of operations. Column 1 presents the disruption resolution scenario, Column 2 the average travel time, Column 3 the average waiting time, Column 4 the average generalized travel time, Column 5 the cycle time, Column 6 the percentage of track occupancy and Column 7 the average output delay. Columns 2-4 only consider the corridor Den Bosch to

Table 1: Performance of the train schedules computed for each scenario.

\begin{tabular}{|c|c|c|c|c|c|c|}
\hline $\begin{array}{l}\text { Dis. Res. } \\
\text { Scenario }\end{array}$ & $\begin{array}{c}\text { Travel } \\
\text { Time (s) }\end{array}$ & $\begin{array}{l}\text { Waiting } \\
\text { Time (s) }\end{array}$ & $\begin{array}{c}\text { Gen. Travel } \\
\text { Time (s) }\end{array}$ & $\begin{array}{c}\text { Min. Cycle } \\
\text { Time (s) }\end{array}$ & $\begin{array}{c}\text { Track } \\
\text { Occ. }(\%)\end{array}$ & $\begin{array}{c}\text { Output } \\
\text { Delay (s) }\end{array}$ \\
\hline Timetable & 2200 & 600 & 3400 & 3600 & 57 & - \\
\hline $12-0-0$ & 2713 & 638 & 4048 & 4252 & 118 & 66 \\
\hline $8-4-0$ & 3537 & 600 & 4737 & 4008 & 111 & 89 \\
\hline $8-0-4$ & 2457 & 900 & 4218 & 3843 & 106 & 53 \\
\hline $4-4-4$ & 3741 & 956 & 5655 & 3600 & 74 & 74 \\
\hline $4-0-8$ & 2709 & 1800 & 6309 & 3600 & 74 & 48 \\
\hline
\end{tabular}


Utrecht and vice versa. All the indicators but the track occupancy are reported in seconds.

The results of Table 1 show that dispatchers have five alternative non-dominated solutions. The solution of scenario $12-0-0$, with no train rerouting and no service cancellation, results in a smaller travel time compared to scenario 8-4-0, with train rerouting. For these two scenarios, the cycle time and the percentage of track occupancy can be considered unacceptable, since putting such a kind of scenario into operations would result in a non-cyclic timetable structure. Differently, the train schedules computed for scenarios 4-4-4 and 4-0-8 are stable, since their cycle time is not larger than one hour and the track occupancy is below $100 \%$. However, the latter two scenarios present the largest generalized travel time compared to the other scenarios, due to the small number of trains scheduled on the disrupted line.

Concerning the other indicators related to passengers' delays, there is a tradeoff between choosing to globally reroute trains (e.g., scenario 8-4-0) and choosing to cancel train services (e.g., scenario 8-0-4). On one hand, the latter scenario presents a smaller travel time compared to the other scenarios and a limited output delay, even if the waiting time for passengers at stations is one half longer than the one in the original timetable. On the other hand, the former scenario presents no increase of the waiting time, while the longer travel time is due to the additional time to run the global rerouting. We observe that the dispatchers' decision would be easier if they know the number of passengers that are travelling for each origindestination pair, since this information can be used to avoid generation of further delays due to overcrowding at stations and on board trains.

\section{Conclusions}

This paper applies an innovative DSS to support the dispatching process in case of disrupted traffic situations. We present an advanced disruption handling procedure that computes feasible train schedules and evaluates alternative resolutions of disrupted situations, such as global rerouting or cancellation of train services. In the computational results, performance indicators assess the negative effect of disruptions on the quality of the railway services. This would allow the dispatchers to choose the most effective disruption resolution scenario and the corresponding microscopic plan of operations for dealing with the disrupted traffic situation.

Future research should address the implementation of a dispatcher user interface, enabling fast and simple communication of key indicators without information overload. Multiple scenarios and dispatching solutions could be automatically generated on the fly, based on the actual infrastructure availability. Such an approach could also be used in the planning stage in order to evaluate the feasibility and performance of alternative timetables. After the timetable assessment, the rolling stock and crew schedules would also have to be updated accordingly. 


\section{Acknowledgements}

We thank ProRail managers for providing the examples. This work is partially supported by the research program TRANSUMO "Reliable Transport Chains" and by the Italian Ministry of Research, Grant number RBIP06BZW8, project FIRB "Advanced tracking system in intermodal freight transportation".

\section{References}

[1] Balcombe, R., Mackett, R., Paulley, R., Preston, J., Shires, J., Titheridge, H., Wardman, M., White, P. (2004) The demand for public transport: a practical guide. TRL Report 593, United Kingdom.

[2] Clausen, J. (2007) Disruption Management in Passenger Transportation from Air to Tracks. Proceedings of the 7th ATMOS Workshop, Sevilla, Spain.

[3] Corman, F., D’Ariano, A. Pacciarelli, D., Pranzo, M. (2009) Centralized versus distributed railway traffic control. Proceedings of the 11th International Conference on Advanced Systems for Public Transport, Hong Kong.

[4] D’Ariano, A., Pacciarelli, D., Pranzo, M. (2007) A branch and bound algorithm for scheduling trains in a railway network. European Journal of Operational Research 183 643-657.

[5] D'Ariano, A. (2008) Improving Real-Time Train Dispatching: Models, Algorithms and Applications. TRAIL Thesis Series T2008/6, The Netherlands.

[6] D’Ariano, A. (2009) Innovative decision support system for railway traffic control. IEEE Intelligent Transportation Systems Magazine 1(4) 8-16.

[7] Hansen, I.A., Pachl, J. (2008) Railway timetable and traffic: Analysis, Modelling and Simulation, Eurail Press, Germany.

[8] Hirai, C., Tomii, N., Tashiro, Y., Kondou, S., Fujimori, A. (2006) An algorithm for train rescheduling using rescheduling pattern description language R. Computers in Railways X, pp. 551-561.

[9] Jespersen-Groth, J., Potthoff, D., Clausen, J., Huisman, D., Kroon, L.G., Maróti, G., Nielsen, M.N. (2009) Disruption Management in Passenger Railway Transportation. Lecture Notes in Computer Science 5868 399-421.

[10] Nielsen, L.K., Kroon, L.G., Maróti, G. (2009) A Rolling Horizon Based Framework for Rolling Stock Rescheduling. Proceedings of the 3rd Internat. Seminar on Railway Operations Modeling and Analysis, Zurich, Switzerland.

[11] Takagi, R., Weston, P.F., Goodman, C.J., Bouch, C., Armstrong, J., Preston, J., Sone, S. (2006) Optimal train control at a junction in the main line rail network using a new object-oriented signalling system model. Computers in Railways X, pp. 479-488.

[12] Törnquist, J. (2005) Computer-based decision support for railway traffic scheduling and dispatching: A review of models and algorithms. Proceedings of the 5th ATMOS Workshop, Palma de Mallorca, Spain.

[13] Wiklund, M. (2007) Serious breakdowns in the track infrastructure - Calculation of the effects on rail traffic. Proceedings of the 2 nd Internat. Seminar on Railway Operations Modeling and Analysis, Hannover, Germany. 